PROCEEDINGS OF THE

AMERICAN MATHEMATICAL SOCIETY

Volume 131, Number 4, Pages 1109-1113

S 0002-9939(02)06761-8

Article electronically published on November 6, 2002

\title{
A CHARACTERIZATION OF QUASICONVEX VECTOR-VALUED FUNCTIONS
}

\author{
JOËL BENOIST, JONATHAN M. BORWEIN, AND NICOLAE POPOVICI
}

(Communicated by N. Tomczak-Jaegermann)

\begin{abstract}
The aim of this paper is to characterize in terms of scalar quasiconvexity the vector-valued functions which are $K$-quasiconvex with respect to a closed convex cone $K$ in a Banach space. Our main result extends a wellknown characterization of $K$-quasiconvexity by means of extreme directions of the polar cone of $K$, obtained by Dinh The Luc in the particular case when $K$ is a polyhedral cone generated by exactly $n$ linearly independent vectors in the Euclidean space $\mathbb{R}^{n}$.
\end{abstract}

\section{INTRODUCTION}

Various generalizations of the classical notion of quasiconvex real-valued function have been given for vector-valued functions, their importance in vector optimization being nowadays recognized (see e.g. 44, [7] or [10] and references therein). Among them, the concept of cone-quasiconvexity, introduced by Dinh The Luc in [9], is of special interest since it can be characterized in terms of convex level sets. Actually, in one case, this property has been taken as the definition of quasiconvexity for vector-valued functions in an early work [6] of Ferro.

In contrast to the simplicity of its definition, cone-quasiconvexity cannot be easily handled from the point of view of scalarization. This is because it is not stable under composition with functionals belonging to the nonnegative polar of the ordering cone, essentially because the sum of quasiconvex functions is usually not quasiconvex, in contrast with the convex case.

For this reason, some authors have restricted their study to the particular class of those cone-quasiconvex functions which satisfy this stability property (the so-called «-quasiconvex functions in [8], or scalarly-quasiconvex functions in [11]). But, the natural way to characterize cone-quasiconvexity via scalar quasiconvexity seems to be that indicated by Dinh The Luc in [10] in the particular case when the ordering cone is generated by an algebraic base of a finite-dimensional space, which consists in imposing the above mentioned stability property just to the extreme directions of the nonnegative polar cone. The primary aim of this paper is to show that this

Received by the editors July 7, 2001.

2000 Mathematics Subject Classification. Primary 26B25; Secondary 90C29.

Key words and phrases. Quasiconvex vector-valued functions, scalarization, polar cones.

The second author's research was supported by NSERC and by the Canada Research Chair Programme.

The third author's research was supported by CNCSIS Romania under Grant no. 1066/2001. 
characterization of cone-quasiconvexity is still true for any closed convex cone with nonempty interior in a Banach space - and indeed somewhat more generally.

\section{Preliminaries}

Throughout we consider a Banach space $Y$, partially ordered by a nonempty closed convex cone $K \subset Y$. As usual, we denote by

$$
K^{+}:=\left\{\ell \in Y^{\star} \mid \ell(y) \geq 0, \forall y \in Y\right\}
$$

the polar cone of $K$ in the topological dual $Y^{\star}$ of $Y$, and by extd $K^{+}$the set of its extreme directions. Recall that $\ell \in \operatorname{extd} K^{+}$if and only if $\ell \in K^{+} \backslash\{0\}$ and for all $\ell_{1}, \ell_{2} \in K^{+}$such that $\ell=\ell_{1}+\ell_{2}$ we actually have $\ell_{1}, \ell_{2} \in \mathbb{R}_{+} \ell$.

The following preliminary result gives a weak lattice property and will play a key role in the proof of our main theorem. For $y, z \in Y$ the notation $y \leq_{K} z$ will mean $z-y \in K$.

Lemma 2.1. Assume that $\left(Y, \leq_{K}\right)$ is directed, i.e.

$$
\text { for all } y_{1}, y_{2} \in Y \text { there is } z \in Y \text { with } y_{1} \leq_{K} z \text { and } y_{2} \leq_{K} z \text {. }
$$

Let $\ell \in \operatorname{extd} K^{+}$and let $y_{1}, y_{2} \in Y$ be such that $\ell\left(y_{1}\right) \leq 0$ and $\ell\left(y_{2}\right) \leq 0$. Then, for every $\varepsilon>0$ there exists $z_{\varepsilon} \in Y$ satisfying $y_{1} \leq_{K} z_{\varepsilon}, y_{2} \leq_{K} z_{\varepsilon}$ and $\ell\left(z_{\varepsilon}\right) \leq \varepsilon$.

Proof. Consider the abstract linear program

$$
\mu:=\inf \left\{\ell(z) \mid z \in Y, z \geq_{K} y_{1}, z \geq_{K} y_{2}\right\},
$$

which can be rewritten as $\mu=\inf \left\{\ell(z) \mid z \in Y, 0 \in\left(y_{1}, y_{2}\right)+H(z)\right\}$, where $H: Y \rightarrow 2^{Y} \times 2^{Y}$ is the convex process defined by $H(z)=(-z+K,-z+K)$ for all $z \in Y$. Since (1) is equivalent to $Y=K-K$, which ensures the surjectivity of $H$, by Corollary 2.1 in [3] it follows that $H$ is open at zero (see [2, 3] for a detailed study of convex processes). Then, by the Lagrange multiplier theorem in 1 there exist $\ell_{1}, \ell_{2} \in Y^{\star}$ such that

$$
\mu \leq \inf \left\{\ell(z)+\ell_{1}\left(y_{1}-z+k_{1}\right)+\ell_{2}\left(y_{2}-z+k_{2}\right) \mid z \in Y \text { and } k_{1}, k_{2} \in K\right\} .
$$

Taking into account that $\mu$ in (2) is finite, we infer that $\ell_{1}, \ell_{2} \in K^{+}$,

$$
\ell=\ell_{1}+\ell_{2} \quad \text { and } \quad \mu \leq \ell_{1}\left(y_{1}\right)+\ell_{2}\left(y_{2}\right) .
$$

Since $\ell \in \operatorname{extd} K^{+}$, we must have $\ell_{1}=t_{1} \ell$ and $\ell_{2}=t_{2} \ell$ for some $t_{1} \geq 0$ and $t_{2} \geq 0$. The hypothesis on $y_{1}$ and $y_{2}$ now shows that

$$
\mu \leq t_{1} \ell\left(y_{1}\right)+t_{2} \ell\left(y_{2}\right) \leq 0 .
$$

This clearly implies the desired conclusion.

Remarks 2.2. 1. It is easy to see that if $\operatorname{int} K \neq \emptyset$, then $\left(Y, \leq_{K}\right)$ is directed (not necessarily a lattice). In this case extd $K^{+}$is nonempty (see Remark 3.2, 1).

2. Note that $\left(Y, \leq_{K}\right)$ may be a lattice and hence directed, with extd $K^{+} \neq \emptyset$, even if int $K=\emptyset$ (see Example 3.3 .3 ).

3. In certain lattice cones, such as $K^{+}=L_{q}^{+}[0,1]$ with $1<q<+\infty$, there are no extreme directions. Of course, in this case, Lemma 2.1 is not relevant.

4. The extremality of $\ell$ in Lemma 2.1 is essential. Indeed consider $Y=\mathbb{R}^{2}$, $K=\mathbb{R}_{+}^{2}$, and let $\ell=(1,1) \in K^{+} \backslash \operatorname{extd} K^{+}$. By choosing $y_{1}=(1,-1)$ and $y_{2}=(-1,1)$, it is easy to see that the conclusion of Lemma 2.1 fails to be true. This explains neatly why in our main Theorem below, (ii) does not usually hold for all members of $K^{+}$. 
5. We could give provisions for the infimum in (2) to be attained. Certainly this holds when $\left(Y, \leq_{K}\right)$ is a lattice, and also when $K$ is a polyhedral cone with nonempty interior in $Y=\mathbb{R}^{n}$ (which is not necessarily a lattice). In this case the conclusion of Lemma 2.1 can be improved in the sense that a $z_{\varepsilon}$ exists with $\ell\left(z_{\varepsilon}\right) \leq 0$.

\section{MAIN RESUlt}

In what follows, $f: D \rightarrow Y$ will denote a function defined on a nonempty convex subset $D$ of a vector space $X$. Recall that $f$ is said to be $K$-quasiconvex if for all $y \in Y$ the level set $\left\{x \in D \mid f(x) \leq_{K} y\right\}$ is convex. We have the following characterization of $K$-quasiconvexity in terms of scalar quasiconvexity:

Theorem 3.1. Assume that $\left(Y, \leq_{K}\right)$ is directed and suppose that

$$
K^{+} \text {is the weak-star closed convex hull of } \operatorname{extd} K^{+} \text {. }
$$

Then the following assertions are equivalent:

(i) $f$ is K-quasiconvex;

(ii) $\ell \circ f$ is quasiconvex for every extreme direction $\ell$ of $K^{+}$.

Proof. Suppose that (ii) holds and fix $y \in Y$. Let $x_{1}, x_{2} \in\left\{x \in D \mid f(x) \leq_{K} y\right\}$ and let $t \in[0,1]$. Then, for every extreme direction $\ell$ of $K^{+}$, we have $\ell\left(f\left(x_{1}\right)\right) \leq \ell(y)$ and $\ell\left(f\left(x_{2}\right)\right) \leq \ell(y)$. As $\ell \circ f$ is quasiconvex, it follows that

$$
\ell\left(f\left(t x_{1}+(1-t) x_{2}\right)-y\right) \leq 0 .
$$

This inequality being true for all extreme directions of $K^{+}$, it follows by (3) that

$$
y-f\left(t x_{1}+(1-t) x_{2}\right) \in K^{++}=K \text {. }
$$

Hence $t x_{1}+(1-t) x_{2} \in\left\{x \in D \mid f(x) \leq_{K} y\right\}$, as required.

Conversely suppose that (i) holds and let $\ell$ be an extreme direction of $K^{+}$. We need to establish that for all $\lambda \in \mathbb{R}$ the level set $D_{\lambda}:=\{x \in D \mid \ell \circ f(x) \leq \lambda\}$ is convex. To this end fix an arbitrary $\lambda \in \mathbb{R}$, and let $x_{1}, x_{2} \in D_{\lambda}$ and $t \in[0,1]$. Since $\ell \in \operatorname{extd} K^{+}$we have $\ell \neq 0$ and hence $\lambda=\ell(y)$ for some $y \in Y$. By applying Lemma 2.1 to $y_{1}:=f\left(x_{1}\right)-y, y_{2}:=f\left(x_{2}\right)-y$, and an arbitrary $\varepsilon>0$, we infer the existence of some $z_{\varepsilon} \in Y$ such that $y_{1} \leq_{K} z_{\varepsilon}, y_{2} \leq_{K} z_{\varepsilon}$ and $\ell\left(z_{\varepsilon}\right) \leq \varepsilon$. Then

$$
f\left(t x_{1}+(1-t) x_{2}\right)-y \leq_{K} z_{\varepsilon}
$$

because $f$ is $K$-quasiconvex. Then

$$
\ell\left(f\left(t x_{1}+(1-t) x_{2}\right)\right) \leq \ell(y)+\ell\left(z_{\varepsilon}\right) \leq \lambda+\varepsilon .
$$

Since $\varepsilon$ was arbitrary we conclude that $t x_{1}+(1-t) x_{2} \in D_{\lambda}$, completing the proof.

Remarks 3.2. 1. If int $K \neq \emptyset$, then $K^{+}$has a bounded hence weak-star compact base and so, by the Krein-Milman Theorem, it follows that condition (3) is fulfilled. Indeed, we take $e \in \operatorname{int} K$ and observe that extreme points of $\left\{\mu \in K^{+} \mid \mu(e)=1\right\}$ yield the desired extreme directions.

2. Under the assumption that $K^{+}$has a weakly compact convex base, the implication (ii) $\Rightarrow$ (i) in Theorem 3.1 was also proven by Dinh The Luc in [10].

3. Of course, condition (3) may hold even if int $K=\emptyset$ (see Example [3.3 3). 
4. When the dual cone has a strictly convex base, then every boundary direction is extreme and so every such composition is quasiconvex. This is the case of the right circular Bishop-Phelps cone. Another interesting case is that of the cone of positive semidefinite matrices in the space of symmetric matrices of given dimension. Their extremal structure is well understood: extreme and exposed directions coincide and correspond to $A \rightarrow \operatorname{tr}(P A)$ for a selfadjoint projection $P$.

Examples 3.3. 1. Let $Y=\mathbb{R}^{n}$ and let $K$ be a polyhedral cone with nonempty interior in $\mathbb{R}^{n}$. Then $K^{+}$is also polyhedral and is generated by some extreme directions, say $d_{1}, \ldots, d_{m}$. In this case, Theorem 3.1 shows that a function $f=$ $\left(f_{1}, \ldots, f_{n}\right): D \rightarrow Y$ defined on a nonempty convex set $D \subset X$ is $K$-quasiconvex if and only if, for all $i \in\{1, \ldots, m\}$, the function $\left\langle d_{i}, f(\cdot)\right\rangle$ is quasiconvex. Note that this characterization was already obtained by Dinh The Luc in [10] for the particular case when $m=n$. Obviously, if $K=\mathbb{R}_{+}^{n}$, then $f$ is $K$-quasiconvex if and only if its scalar components $f_{1}, \ldots, f_{n}$ are quasiconvex.

2 . Let $\Omega$ be a nonempty compact Hausdorff topological space. Consider the Banach space $Y=C(\Omega)$ of real continuous functions on $\Omega$, equipped with the usual uniform norm. Then $K=\{y \in C(\Omega) \mid \forall t \in \Omega, y(t) \geq 0\}$ is a closed convex cone with nonempty interior. The dual space $C(\Omega)^{\star}$ being the space of Radon measures, the polar cone $K^{+}$is the cone of positive Radon measures (see e.g. [5]). Its extreme directions are the Dirac measures multiplied by strictly positive numbers. By virtue of Theorem [3.1, a function $f: D \rightarrow C(\Omega)$ is $K$-quasiconvex if and only if the function $x \in D \longmapsto f(x)(t) \in \mathbb{R}$ is quasiconvex, for every $t \in \Omega$.

3. Let $Y=l^{p}(1 \leq p<+\infty)$ and $K=l_{+}^{p}=\left\{y=\left(y_{i}\right)_{i \in \mathbb{N}} \in l^{p} \mid \forall i \in \mathbb{N}, y_{i} \geq 0\right\}$. Then, by identifying $\left(l^{p}\right)^{\star}$ with $l^{q}(1 / p+1 / q=1)$, we have $K^{+}=l_{+}^{q}$. Note that in this case the interior of $K$ is empty while condition (31) holds. Theorem 3.1 shows that a function $f=\left(f_{i}\right)_{i \in \mathbb{N}}: D \rightarrow l^{p}$ is $K$-quasiconvex if and only if $f_{i}$ is quasiconvex for all $i \in \mathbb{N}$.

\section{REFERENCES}

1. J. M. Borwein, A Lagrange multiplier theorem and a sandwich theorem for convex relations, Math. Scand. 48 (1981), no. 2, 189-204. MR 83d:49027

2. _ Adjoint process duality, Math. Oper. Res. 8 (1983), no. 3, 403-434. MR 85h:90092

3. Norm duality for convex processes and applications, J. Optim. Theory Appl. 48 (1986), no. 1, 53-64. MR 87d:90126

4. A. Cambini and L. Martein, Generalized concavity in multiobjective programming, Generalized convexity, generalized monotonicity: recent results (Luminy, 1996), Kluwer Acad. Publ., Dordrecht, 1998, pp. 453-467. MR 99g:90105

5. G. Choquet, Lectures on analysis. Vol. I: Integration and topological vector spaces, Vol. II: Representation theory, W. A. Benjamin, Inc., New York-Amsterdam, 1969. MR 40:3252, MR 40:3253

6. F. Ferro, Minimax type theorems for n-valued functions, Ann. Mat. Pura Appl. (4) 132 (1982), 113-130. MR 84i:49045

7. J. Jahn, Mathematical vector optimization in partially ordered linear spaces, Verlag Peter D. Lang, Frankfurt am Main, 1986. MR 87f:90095

8. V. Jeyakumar, W. Oettli, and M. Natividad, A solvability theorem for a class of quasiconvex mappings with applications to optimization, J. Math. Anal. Appl. 179 (1993), no. 2, 537-546. MR 94i:90094

9. D. T. Luc, Connectedness of the efficient point sets in quasiconcave vector maximization, J. Math. Anal. Appl. 122 (1987), no. 2, 346-354. MR 88f:90154 
10. Theory of vector optimization, Springer-Verlag, Berlin, 1989. MR 92e:90003

11. P. H. Sach, Characterization of scalar quasiconvexity and convexity of locally Lipschitz vectorvalued maps, Optimization 46 (1999), no. 3, 283-310. MR 2001d:90069

LACO, UPRESSA 6090, Department of Mathematics, University of Limoges, 87060 Limoges, France

E-mail address: benoist@unilim.fr

Department of Mathematics, Simon Fraser University, Burnaby, British Columbia, CANADA V5A 1S6

E-mail address: borwein@cecm.sfu.ca

Faculty of Mathematics and Computer Science, Babeş-Bolyai University of Cluj, 3400 Cluj-Napoca, Romania

E-mail address: popovici@math.ubbcluj.ro 